\title{
INTERPRETACIÓN DE LAS ENCUESTAS DE OPINION SOBRE LA INTENCIÓN DE VOTO EN LAS ELECCIONES GENERALES EN ESPAÑA (1977-2000)
}

\section{INTERPRETATION OF OPINION SURVEYS ON THE INTENTION TO VOTE IN SPANISH GENERAL ELECTIONS}

(1977-2000)

\section{AUTORES}

Yanet Acosta Meneses

Universidad Complutense de Madrid, España.

yacosta33@gmail.com

\section{Luis Varona Aguado}

Universidad de Zaragoza. Zaragoza (España).

Ivarona@unizar.es

\section{RESUMEN}

Las encuestas de opinión, publicadas en la prensa escrita, para conocer la intención de voto ante unas elecciones generales han sido parte del debate político. La interpretación de los datos, tanto por parte de los periodistas como de las propias empresas que las elaboran, son cruciales para entender lo polémicas que han llegado a ser en muchas ocasiones. Este artículo estudia el grado de certeza de estas interpretaciones a través de análisis cuantitativos y cualitativos de las encuestas publicadas en los principales diarios, en las elecciones generales españolas, desde 1977 hasta el año 2000.

\section{PALABRAS CLAVE}

Encuestas - Intención de voto - Prensa escrita - Elecciones generales. 


\section{ABSTRACT}

Opinion polls published in newspapers to find out the intention to vote at general elections have been part of political debate. The data interpretation by both journalists and companies themselves that are developed, are crucial to understand the controversies that have become at several times. This article studies the degree of certainty of these interpretations, through quantitative and qualitative analysis of surveys published in major newspapers, in Spanish general elections from 1977 to 2000.

\section{KEY WORDS}

Polls - Voting intention - Newspapers - General elections.

\section{ÍNDICE}

1. Introducción.

2. Metodología.

2.1 Método cuantitativo.

2.2 Método cualitativo.

3. El devenir de las empresas.

3.1 Las elecciones de 1977.

3.2 Las elecciones de 1979.

3.3 Las elecciones de 1982.

3.4 Las elecciones de 1986.

3.5 Las elecciones de 1989.

3.6 Las elecciones de 1993.

3.7 Las elecciones de 1996.

3.8 Las elecciones de 2000.

4. Una visión longitudinal de los sondeos. 
4.1 Sesgo de la intención de voto al PSOE.

4.2 Sesgo de la intención de voto al PP.

5. Conclusiones.

6. Bibliografía.

7. Hemerografía.

8. Webgrafía.

\section{Introducción}

Las encuestas de opinión, cuyo origen data los años 20 con los trabajos de Gallup, tratan de verificar un resultado contrastable, los verdaderos resultados electorales. Sin embargo, la controversia acerca de las encuestas se basa en la obtención de datos y en la interpretación de los resultados, todo depende de la empresa que las realice y del periodista que escriba acerca de ellas.

Gallup obtuvo gran éxito con sus encuestas, pero el mal sabor de boca que había dejado el experimento de los votos de paja (ahora renovado con las encuestas que se realizan en sitios web) se recuperó en 1936, con una encuesta catastrófica que al Digest le mereció titular "What went wrong with the polls?" (¿Qué salió mal en las urnas?"). En esta encuesta, daban como ganador a Alfred M. Landon, en lugar del victorioso Franklin Delano Roosvelt.

En España, las encuestas de opinión llegan a los medios de comunicación en 1977, con las primeras elecciones generales en democracia tras la muerte de Franco. La importancia que los medios de comunicación conceden a las encuestas van in crescendo desde ese momento y hasta las elecciones del 12 de marzo de 2000. 
En este periodo, las encuestas de opinión gozaron de más o menos credibilidad dependiendo de su grado de acierto. En 1982, las encuestas acertaron de lleno, pese a la incredulidad tanto de la interpretación de la prensa como de los lectores. Sin embargo, el gran fiasco de los datos obtenidos de las encuestas de opinión en las elecciones generales de 1993 y 1996, fomentó la polémica. La interpretación de los datos realizada por estadísticos y periodistas no fueron las más certeras.

Este fracaso en el análisis ha provocado un escepticismo en la sociedad que ha llevado a planteamientos como "yo no creo en las encuestas", como si de una religión se tratase. En este trabajo, se hará un repaso histórico de las encuestas publicadas en los medios de comunicación en referencia a las elecciones generales.

Esta evolución se analizará cualitativa y cuantitativamente. En lo que se refiere al análisis cualitativo, se hará una recopilación de los artículos interpretativos de las encuestas, así como de los artículos de opinión y editoriales acerca del tema. El análisis cuantitativo tiene como objetivo valorar el grado de ajuste de estas encuestas con respecto a los resultados electorales. De esta forma se puede analizar con un método contrastado el grado de acercamiento de los sondeos de opinión a la realidad objetiva que ofrecen los resultados electorales.

Con estos datos objetivos, se puede situar en paralelo las opiniones y los datos estadísticos. Así, obtendremos una constatación estadística de las opiniones e interpretaciones plasmadas en los medios de comunicación.

\section{Metodología}

Para realizar este trabajo se ha acotado el universo a analizar, con el objetivo de poder realizar un estudio en mayor profundidad. Así, las encuestas de opinión 
examinadas son las últimas que se publican antes de las elecciones generales. Las elecciones seleccionadas son las de carácter general, por su importancia en la política del Estado, en la opinión pública y en los medios de comunicación. Los periódicos escogidos son los dos de mayor importancia, ya sea por su difusión o por su interés, a nivel nacional: Diario 16 y El País hasta 1989, y El Mundo y El País, a partir de 1989. Para llevar a cabo este trabajo se utilizarán dos métodos de análisis, uno cuantitativo y otro cualitativo. Para llevar a cabo cada uno de ellos se utilizarán unas herramientas que a continuación se detallan.

\subsection{Método cuantitativo}

La metodología utilizada para el análisis cuantitativo se ha realizado haciendo uso de procedimientos estadísticos sencillos. Un sondeo de opinión consiste en una estrategia estadística para obtener información sobre un determinado tema en una población cualquiera. En concreto, un sondeo electoral investiga las opciones electorales de la población constituida por los electores que acuden a votar. Esta población se define como la población objeto del estudio. Dada la imposibilidad de realizar un cuestionario a todos y cada uno de los componentes de esta población, se obtiene una muestra de individuos, sobre los cuales se realiza el trabajo de campo.

La selección de la muestra de individuos es un elemento clave para la consecución del objetivo de la encuesta y se trata, seguramente, del proceso más complicado con el que tiene que encontrarse la empresa o persona que diseña la encuesta. Los individuos seleccionados deben constituirse en una muestra representativa de la población objeto. Para ello, los sondeos de opinión electoral que habitualmente se publican en los medios de comunicación, utilizan un muestreo estratificado polietápico. 
Un muestreo estratificado es aquel en el que los elementos de la población se dividen en clases o estratos, y la muestra se toma asignando un número determinado de miembros a cada estrato. Un muestreo se define como polietápico cuando la asignación en estratos no se limita a una única subdivisión, sino que se realizan en etapas sucesivas. Por ejemplo, en un sondeo electoral, la población objeto son todos los votantes del país que, en primer lugar y proporcionalmente, se clasifican en comunidades autónomas, provincias, grupos de edad, sexo, clase social.

Una vez obtenida la muestra, se realiza la encuesta que, normalmente, consiste, por una parte, en una cuestión acerca de la intención de voto directo del encuestado y, por otra, en un número de preguntas que ayuda a definir la orientación política de quienes no definen claramente su preferencia. Posteriormente, los datos son sometidos a un tratamiento estadístico que proporciona los estimadores y los intervalos de confianza que más tarde serán publicados. Este proceso también es un elemento clave en cuanto a la adecuación de la encuesta a los resultados finales, porque las empresas demoscópicas utilizan técnicas correctoras basadas en la experiencia previa, que, si no son adecuadas, pueden incrementar los sesgos y errores cometidos en la predicción.

La encuesta electoral es un caso único en el mundo de los sondeos de opinión, puesto que "la encuesta verdadera" se realiza a los pocos días de los sondeos y, excepcionalmente, se conoce exactamente cuál es la población objeto; eso sí, a posteriori. En este trabajo, se han realizado cuatro análisis cuantitativos diferentes con los resultados de las encuestas. Primero, se ha realizado un estudio de estadística descriptiva con los resultados observados en las encuestas y los resultados esperados en las mismas. Esta operación se calcula con una regla de estricta proporcionalidad a partir de los resultados del día de las elecciones. También se ha llevado a cabo un estudio de ajuste no paramétrico entre los resultados 
observados y los resultados esperados, mediante un cálculo de distancia basado en el contraste de chi-cuadrado, que utiliza la siguiente expresión:

$$
\chi^{2}=\sum_{i=1}^{n c} \frac{\left(O_{i}-E_{i}\right)^{2}}{E_{i}}
$$

Donde nc es el número de clases consideradas en el análisis (partidos políticos en este caso), $\mathrm{O}_{\mathrm{i}}$ es el número de datos observados en la clase $\mathrm{i}$ en el sondeo de opinión y $\mathrm{E}_{\mathrm{i}}$ es el número de datos esperados en la clase i extrapolados del resultado electoral definitivo.

Además, se ha analizado el grado de sesgo de las encuestas a través del cálculo de los cocientes entre los porcentajes de votos esperados y los observados en las encuestas. Con esta operación, se puede saber si los resultados han sido sobrevalorados o infravalorados en las encuestas. Así, un cociente superior a uno, indica una subestimación de los resultados electorales por parte del sondeo y un cociente inferior a uno indica una sobrevaloración de los resultados.

\subsection{Método cualitativo}

El método cualitativo se basa en el análisis de las interpretaciones de los datos realizadas por los periodistas tanto en informaciones como en artículos y editoriales. En este análisis, se identificará la intencionalidad del medio y la influencia del contexto social y político en el que se realizan y publican estas encuestas de opinión. Estos análisis cualitativos se contrastan con los datos objetivos que proporcionan los análisis estadísticos realizados sobre el grado de ajuste de estas encuestas con respecto a los resultados electorales. 


\section{El devenir de las encuestas}

Las encuestas de opinión fueron publicadas en la prensa escrita de distribución nacional desde las primeras elecciones generales en democracia en España tras la muerte de Franco, celebradas en 1977. Desde ese año y hasta el 2000, fecha en la que se fija el final del periodo de estudio, las encuestas de opinión han ido adquiriendo más protagonismo en los diarios. Para su elaboración, empresas especializadas en demoscopia han ido ajustando cada vez más sus índices de corrección para conseguir resultados más cercanos a los reales.

No obstante, la interpretación de los datos en la prensa y en las propias empresas que elaboran las encuestas ha dependido, en gran medida, de la situación política y económica del país en cada momento concreto.

\subsection{Las elecciones de 1977}

Entre la sociedad española de 1977, se prodiga la ilusión tras el referéndum sobre la Reforma Política y la perspectiva de las primeras elecciones democráticas desde febrero de 1936. Sin embargo, también hay miedo entre los españoles, ya que el proceso de transición política se encuentra en su momento más crítico. Entre el 15 de diciembre de 1976 y hasta el 15 de junio de 1977, fecha de celebración de las elecciones, España vive diversos conflictos laborales y sociales, el azote del terrorismo, la legalización de los partidos, el resurgir de los nacionalismos y una crisis económica en ciernes.

En medio de este caos, en febrero de 1977, desaparecen los requisitos más restrictivos para la legalización de los partidos, que culmina el 9 de abril de 1977, el Sábado Santo Rojo, el Gobierno decide la desaparición del Movimiento, el partido único franquista, y legaliza al Partido Comunista de España y, dos días después al 
PSUC (Partit Socialista Unificat de Catalunya), lo que provocó la dimisión instantánea del Almirante Pita de Veiga, Ministro de la Marina.

El Gobierno convoca elecciones para el 15 de junio y surge la sopa de letras o la guerra de siglas, un gran número de partidos políticos concurren a las elecciones. Entre los partidos que se presentan a nivel nacional, de derecha a izquierda, Alianza Popular -liderada por Manuel Fraga-, Unión Centro Democrático -en torno a Adolfo Suárez-, Democracia Cristiana -el gran fracaso de Joaquín Ruiz Giménez-, Partido Socialista Obrero Español -con Felipe González al frente-, el Partido Socialista Popular de Enrique Tierno Galván y José Bono, y el Partido Comunista de España -con los históricos Santiago Carrillo y la Pasionaria. A la izquierda del PCE se sitúa un mosaico de fuerzas (PTE, ORT, LCR, MC), que intenta atraer el voto de quienes no comparten el sentido de la moderación que reina en el PCE y en Comisiones Obreras.

Sin embargo, es el Partido Socialista Obrero Español (PSOE) es el que va a recoger el masivo voto de izquierdas. Tras la muerte de Franco, consigue dar una imagen de juventud, de dinamismo, de capacidad de organización, de aceptación internacional, que hace que la mayoría de la población acabe identificándolo con la oposición al régimen. A la derecha está Alianza Popular (AP), el partido en el que Manuel Fraga Iribarne, brillante alto funcionario del régimen anterior, reúne a todos sus colaboradores, ex franquistas convencidos: Gonzalo Fernández de la Mora, Cruz Martínez Esteruelas, Silva Muñoz, Licinio de la Fuente, Enrique Thomas de Carranza. La extrema derecha está integrada por Fuerza Nueva (FN) de Blas Piñar, y Falange Española de las JONS. Estos dos partidos, que no obtienen representación parlamentaria, recomiendan votar a $A P$ en aquellas provincias en las que no presentan candidaturas.

En medio de este escenario, se sitúa la Unión de Centro Democrático (UCD) de Adolfo Suárez. Se trata de una coalición de partidos entre los que se encuentran los siguientes: Partido Demócrata Cristiano (Álvarez de Miranda), Partido 
Socialdemócrata (Fernández Ordóñez), Unión Socialdemócrata (Eurico de la Peña), Partido Popular (Pío Cabanillas) y Federación de Partidos Demócratas y Liberales (J oaquín Garrigues Walker), entre otros.

En este contexto los sondeos de opinión hacen su aparición en la historia democrática de España. Los resultados de las encuestas de opinión sobre la intención de voto de El País y Diario 16 predicen bien la victoria de UCD, pero infravaloran el voto de la izquierda, sobre todo El País con respecto al PSOE. Las elecciones las gana la UCD por mayoría relativa y las urnas arrojan varias sorpresas: el PSOE arrasa y le quita muchos votos al PCE, mientras que AP, que ha hecho una larga y carísima campaña electoral, obtiene unos resultados muy modestos. Los resultados electorales se hacen esperar varios días debido a la inexperiencia en el escrutinio de los votos.

En el siguiente gráfico, se pueden observar las diferencias entre las encuestas de opinión de estos dos diarios y los resultados electorales:

\section{ELECCIONES 1977}

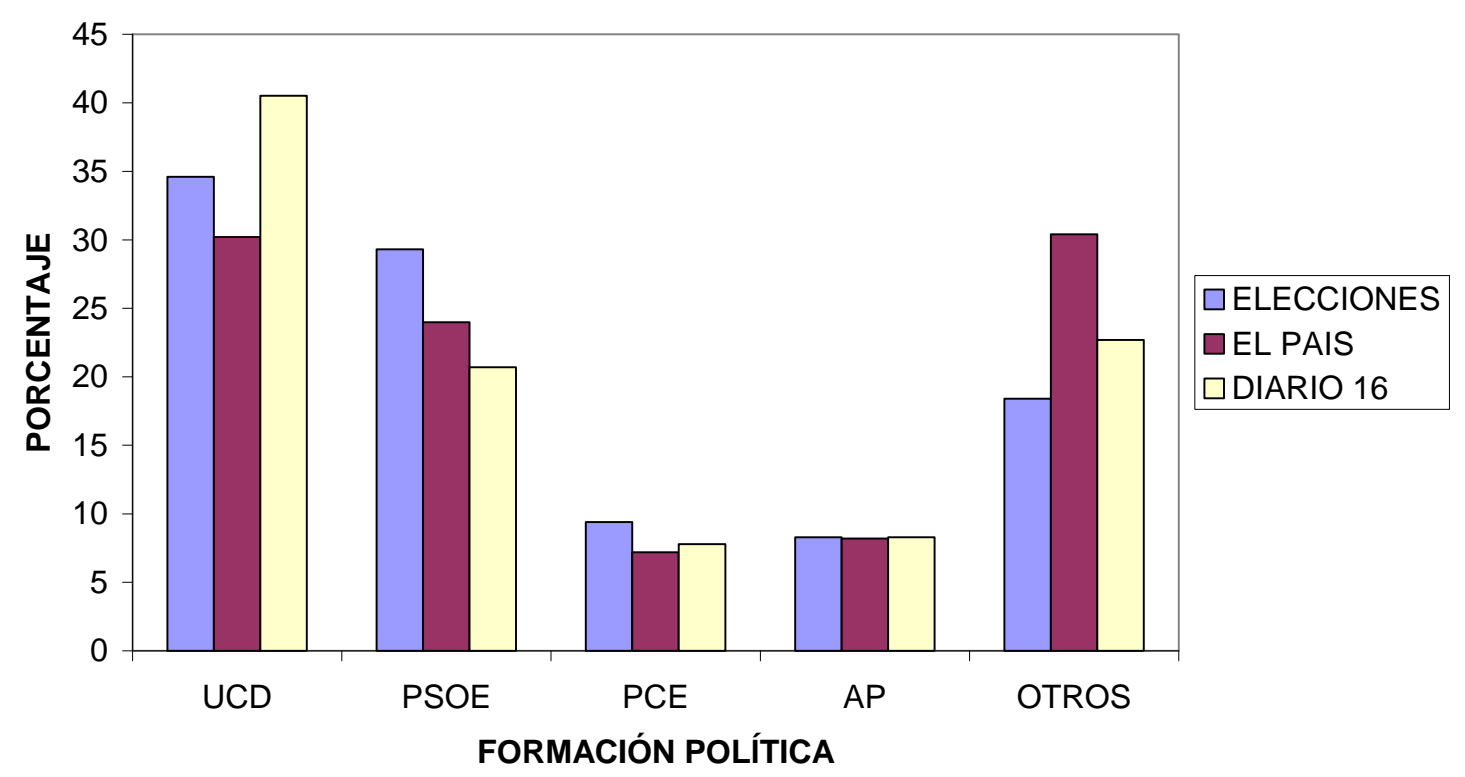


Las tendencias de las encuestas de El País y Diario 16 son similares, ya que ambas dan por ganador a la UCD. Sin embargo, Diario 16 sobreestima los resultados para el partido de centro, mientras que El País los infravalora. En el caso del PSOE, los dos diarios infravaloran la intención de voto y ambos coinciden en interpretar una mayor dispersión del voto entre partidos que la que resultó finalmente. Con respecto al PCE, las encuestas de opinión de ambos diarios también se quedaron por debajo de los resultados finales. Este desajuste se puede achacar a la situación política y el temor del votante a identificarse con la ideología comunista. El análisis demoscópico no consiguió corregir este fenómeno a la hora de interpretar los datos.

\subsection{Las elecciones de 1979}

Para las elecciones de 1979, la Unión de Centro Democrático y el Partido Socialista Obrero Español, tras absorber al partido Socialista Popular, se perfilan en las encuestas de opinión de los dos diarios de información general con mayor circulación en la época como máximos candidatos a la victoria. Mientras, Alianza Popular y el Partido Comunista se presentan como las minorías representativas a nivel nacional.

Los sondeos de opinión ofrecen resultados contradictorios con victorias socialista ( $E$ / País) y centrista (Diario 16). La posterior victoria centrista puso de manifiesto una infraestimación del voto de centro en las encuestas. En estas encuestas se percibe el cambio de tendencia en la población en cuanto a manifestar su intención de voto socialista. Diario 16 prevé resultados superiores a los que realmente se materializaron para el PSOE y tanto este diario como El País se quedan cortos, especialmente este último, en la estimación de voto a UCD. 


\section{ELECCIONES 1979}

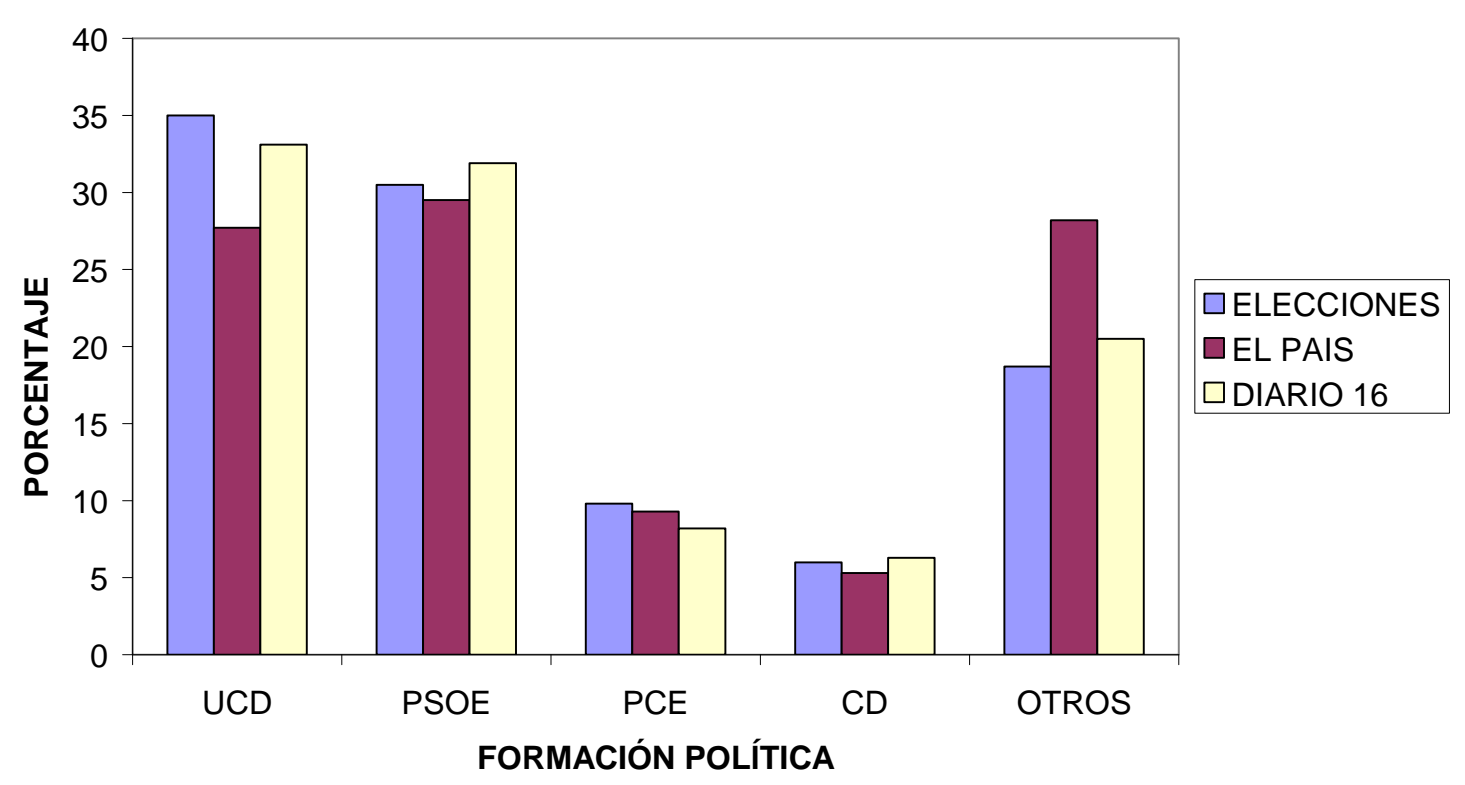

\subsection{Las elecciones de 1982}

Las encuestas de intención de voto publicadas por Diario 16 y El País, para las elecciones convocadas para el 15 de junio de 1982, predicen certeramente la victoria del PSOE y el desastre electoral de la UCD. No obstante, infravaloran los resultados de Alianza Popular (denominada en este momento Coalición Popular) y sobrestiman los pobres resultados del PCE. En el caso de la UCD, Diario 16 sobrevalora el resultado que puede obtener el partido en el Gobierno, mientras que El País lo ajusta bastante bien. Por el contrario, los resultados predichos por ambas encuestas para el PCE son sobrevalorados.

En las encuestas de opinión, El País comete el error de dar mucha importancia a la intención de voto a partidos minoritarios, que posteriormente no se confirma con los resultados de las urnas. 


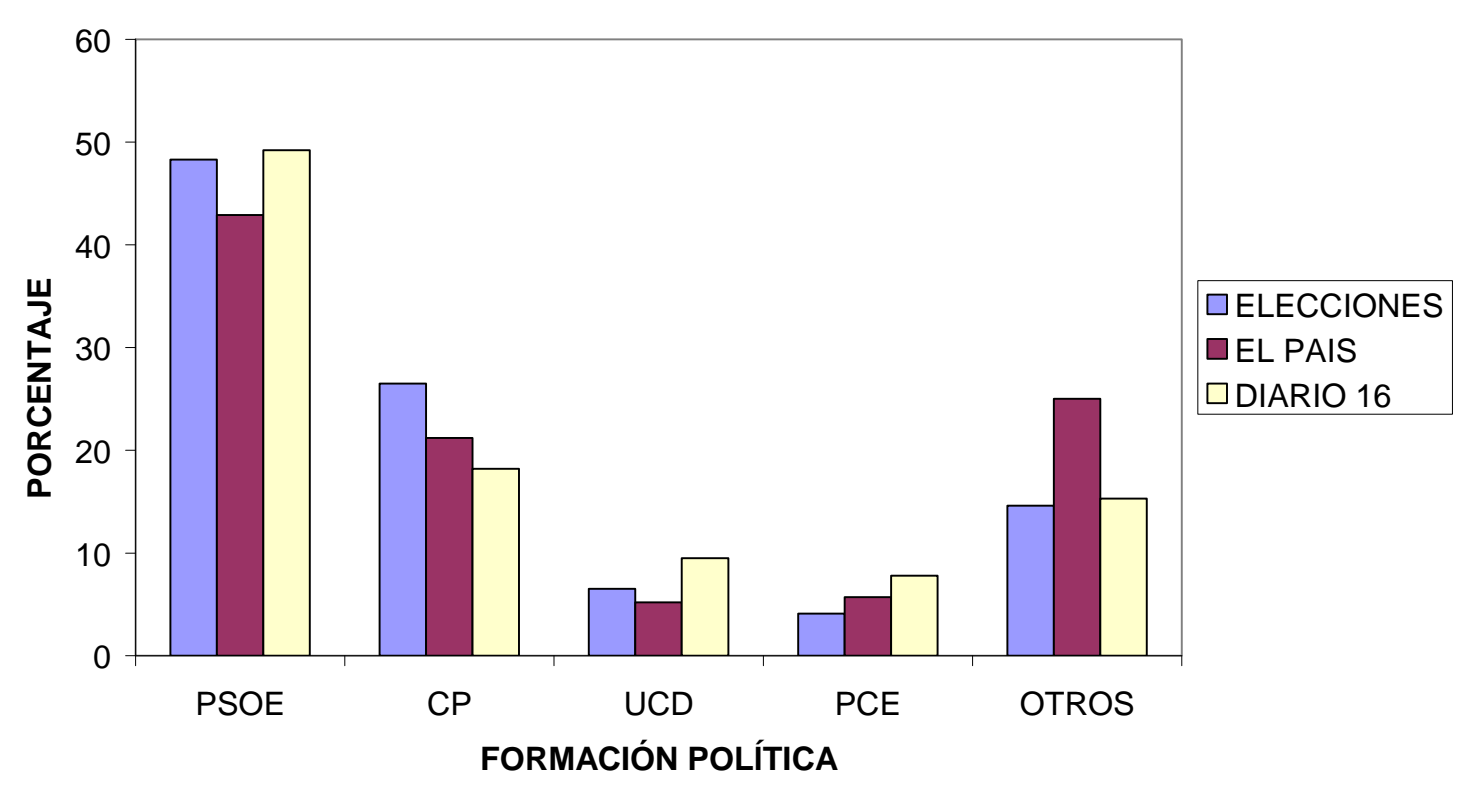

\subsection{Las elecciones de 1986}

Las encuestas de opinión predijeron bien el voto socialista, aunque en ambos diarios se infravaloró el voto a Alianza Popular, partido que arrastra aún su relación con el régimen anterior. El voto al CDS fue sobreestimado por los dos diarios y el del PRD se sobrevalora en El País. Diario 16 predice un mayor voto socialista del real y El País un voto inferior al que luego aparecerá. La Coalición Popular obtiene datos inferiores en ambas encuestas, mientras que CDS e Izquierda Unida obtienen más de lo que realmente consiguieron: 


\section{ELECCIONES 1986}

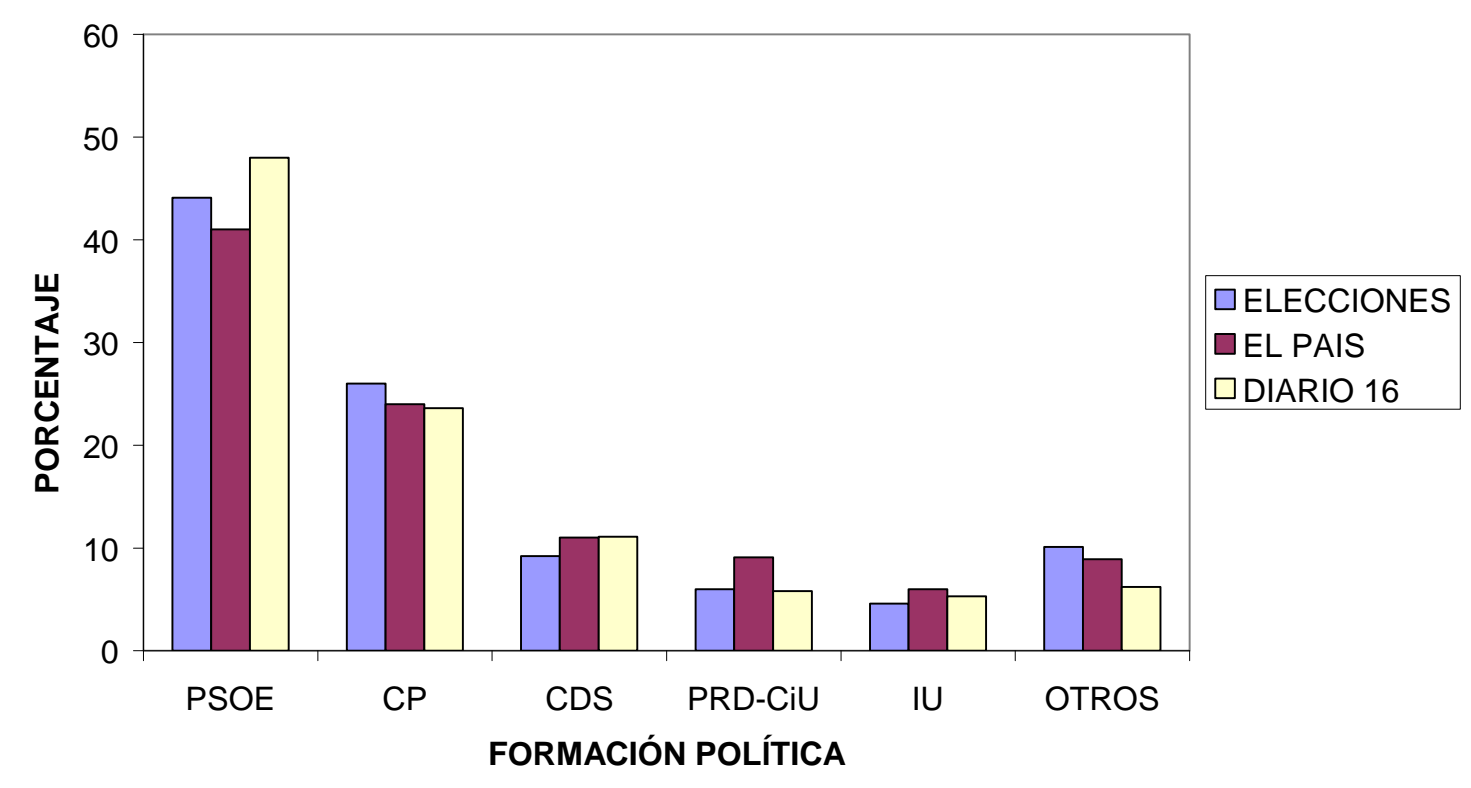

\subsection{Las elecciones de 1989}

En las elecciones de 1989, las encuestas de opinión obtienen su mejor éxito, pues consiguen el mejor ajuste. Tan sólo cabe destacar una ligera subestimación del Partido Popular. El voto socialista está muy bien ajustado tanto en El País como en El Mundo y hay una subestimación del voto Popular, compensada con la sobrevaloración del voto al CDS. En el caso de IU, la intención de voto sigue siendo infravalorada. 


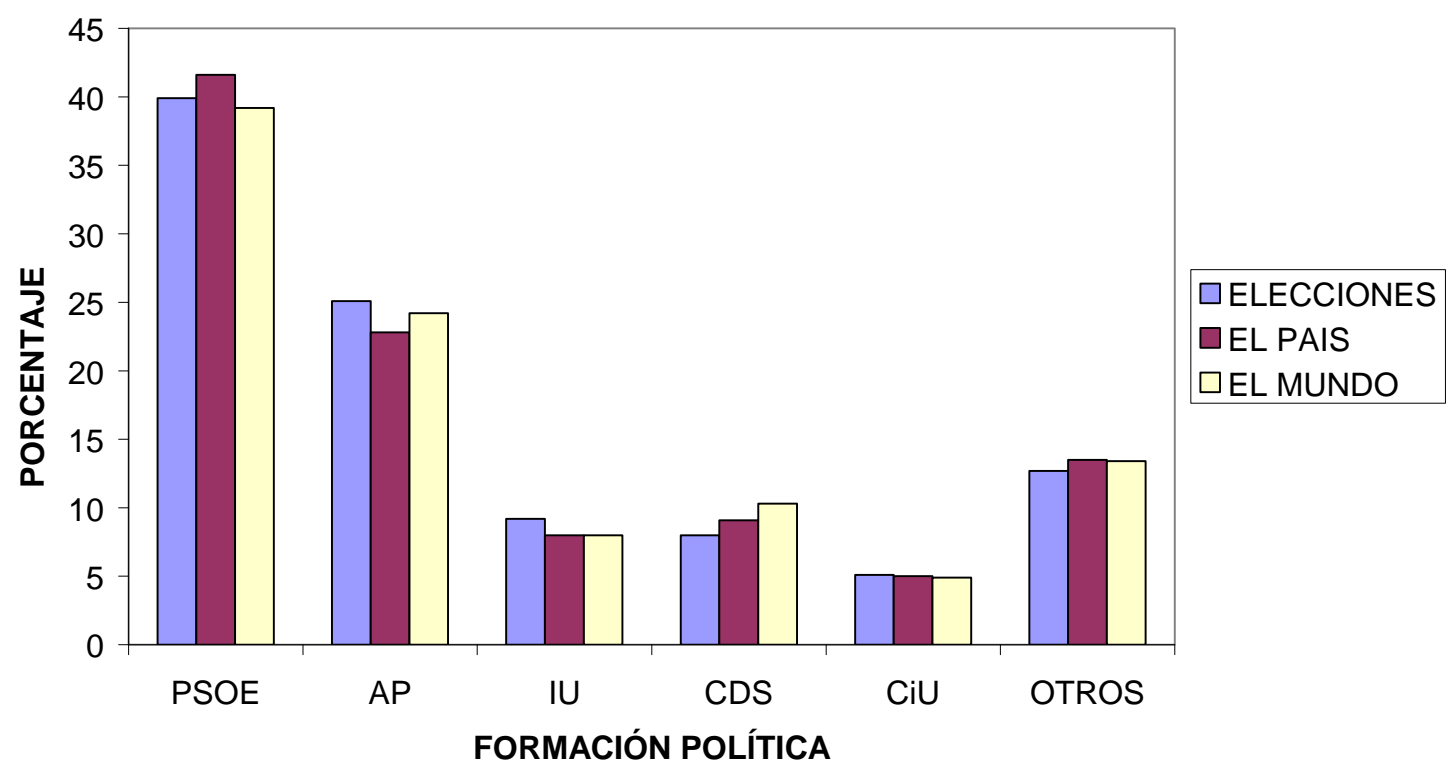

\subsection{Las elecciones de 1993}

Las encuestas de opinión de las elecciones de marzo de 1993 son el gran fracaso de los sondeos modernos porque, aunque cuantitativamente los cálculos son mejores que los del año 1982, cualitativamente se equivocan al predecir que el PP saldría victorioso. En esta ocasión, debido a la situación política, se detecta la ocultación de la intención de voto del electorado socialista y una sobrevaloración al voto para Izquierda Unida. Las dos encuestas fallan en este punto, ya que no acierta a corregir esta ocultación de voto en sus interpretaciones de los datos: 


\section{ELECCIONES 1993}

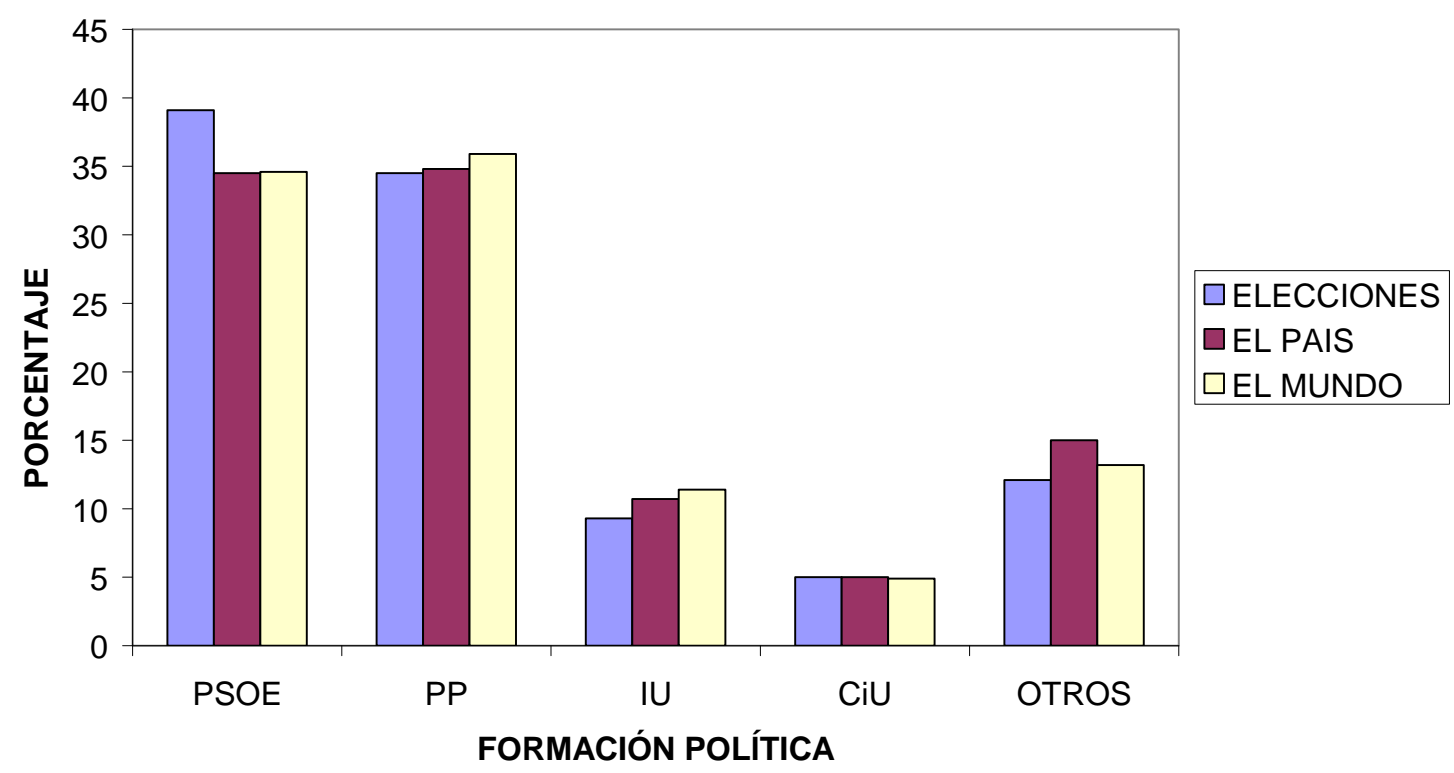

\subsection{Las elecciones de 1996}

Pese a la recuperación económica, las elecciones del 96 se presentaron más igualadas que nunca y con ventaja, según los sondeos para el PP. En las encuestas, el PP ganaba con una diferencia espectacular. Aunque el día de las elecciones, la ventaja del PP fue de apenas un punto. El error de las encuestas es superior al acaecido en 1993. Sin embargo aciertan quién gana, con lo que el error no es de bulto: 

encuestas de opinión sobre la intención de voto en las elecciones generales en España (1977-2000) No 13. Noviembre. Año X. Páginas: 78-101

ISSN: 1576-3420 DOI: http://dx.doi.org/10.15198/seeci.2006.13.78-101

\section{ELECCIONES 1996}

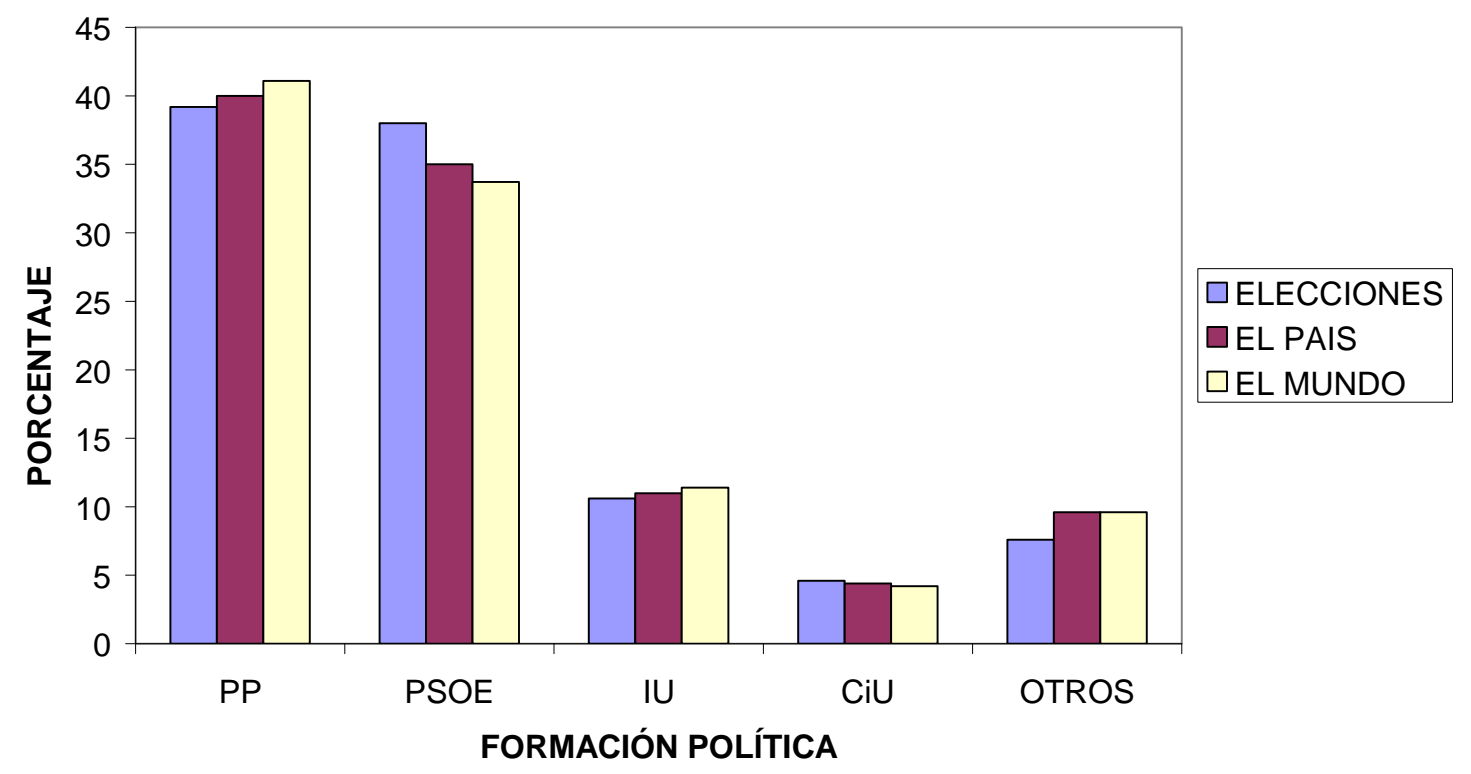

3.8. Las elecciones de 2000

El PP es infravalorado y el PSOE sobrevalorado por las encuestas sobre las elecciones de 2000. Tanto El Mundo como El País fueron incapaces de predecir la mayoría absoluta del PP, mientras que sobrevaloraron a IU: 


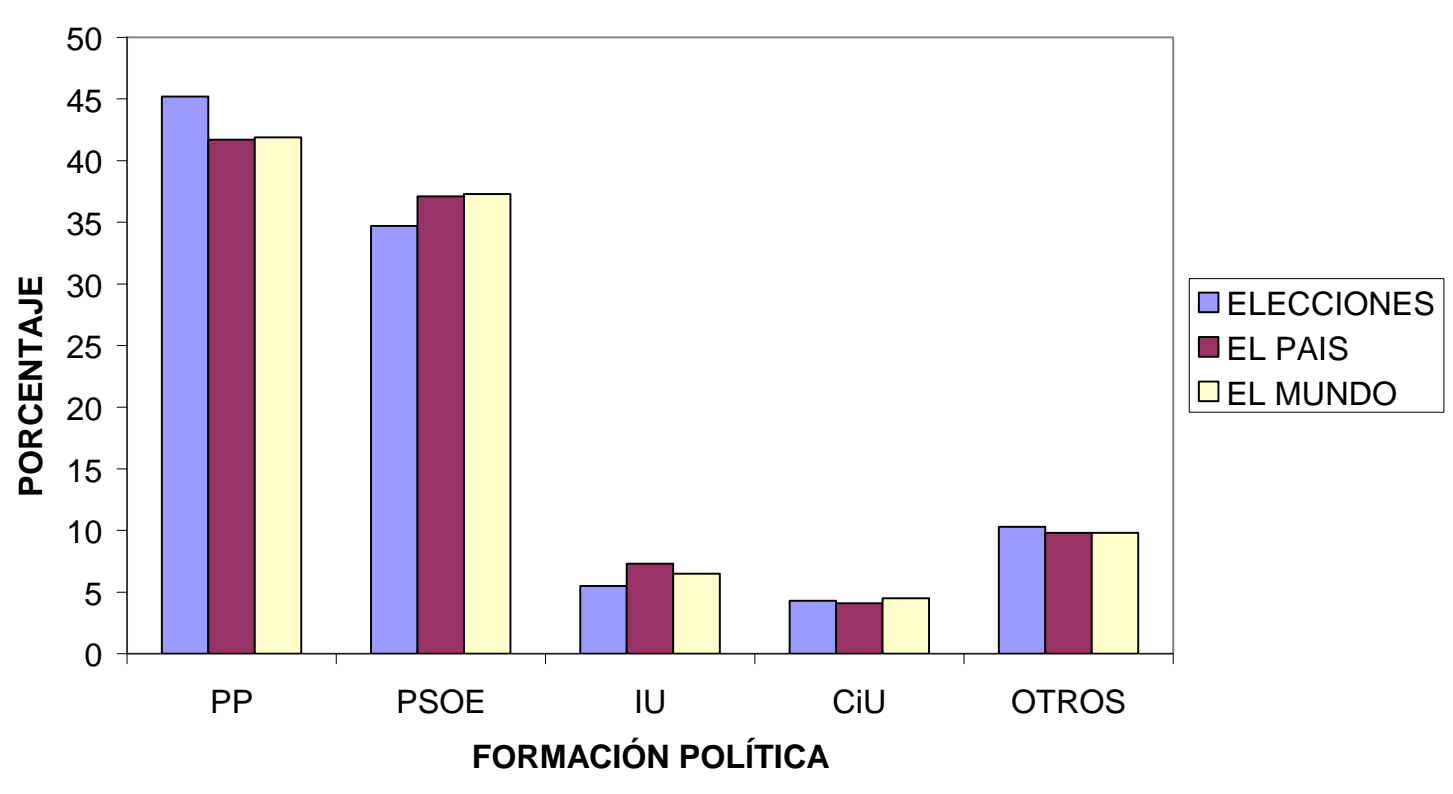

\section{Una visión longitudinal de los sondeos}

En este apartado, se analiza la medida de distancia basada en chi cuadrado entre los sondeos publicados por los diarios estudiados y los resultados electorales. Como se puede observar en la siguiente figura, la calidad de la encuestas ha mejorado considerablemente, especialmente a partir del año 1989: 


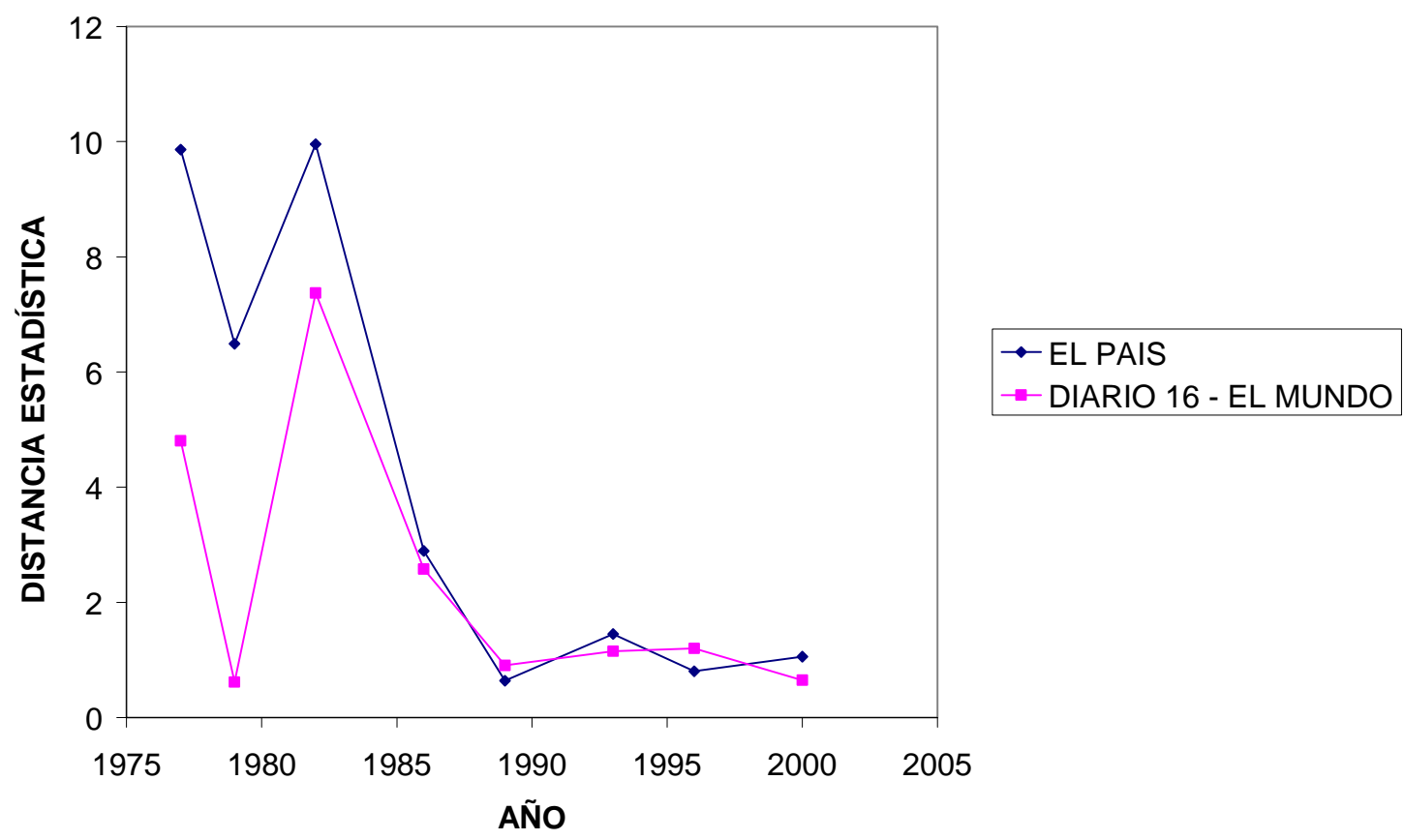

Sin embargo, aunque la calidad de las encuestas ha ido mejorando desde los años 90, es precisamente a partir de esta época, cuando más se critican los análisis demoscópicos publicados en los diarios. La desconfianza hacia estas encuestas se fomentó con el error de las publicadas en el año 1993, que erraron en la predicción del partido ganador. Sin embargo, el error fue de interpretación de los sesgos o de la manera de ocultar la verdadera intención de voto del encuestado.

Se debe tener en cuenta que cuando la diferencia entre las dos principales fuerzas políticas es inferior al error de muestreo de los sondeos o incertidumbre esperada en las encuestas, el resultado debería ser considerado como un "empate técnico". No obstante, se produce una inercia que les lleva a optar por una opción u otra, en función de lo que más se repite en el ambiente mediático. 


\subsection{Sesgo de la intención de voto al PSOE}

En 1977, el PSOE está subestimado pues la salida de la dictadura hace que el votante socialista oculte su voto. De 1979 a 1989, los sesgos son mínimos, pero hay una divergencia dependiendo del medio del que se trate:

EVOLUCIÓN SESGOS PSOE

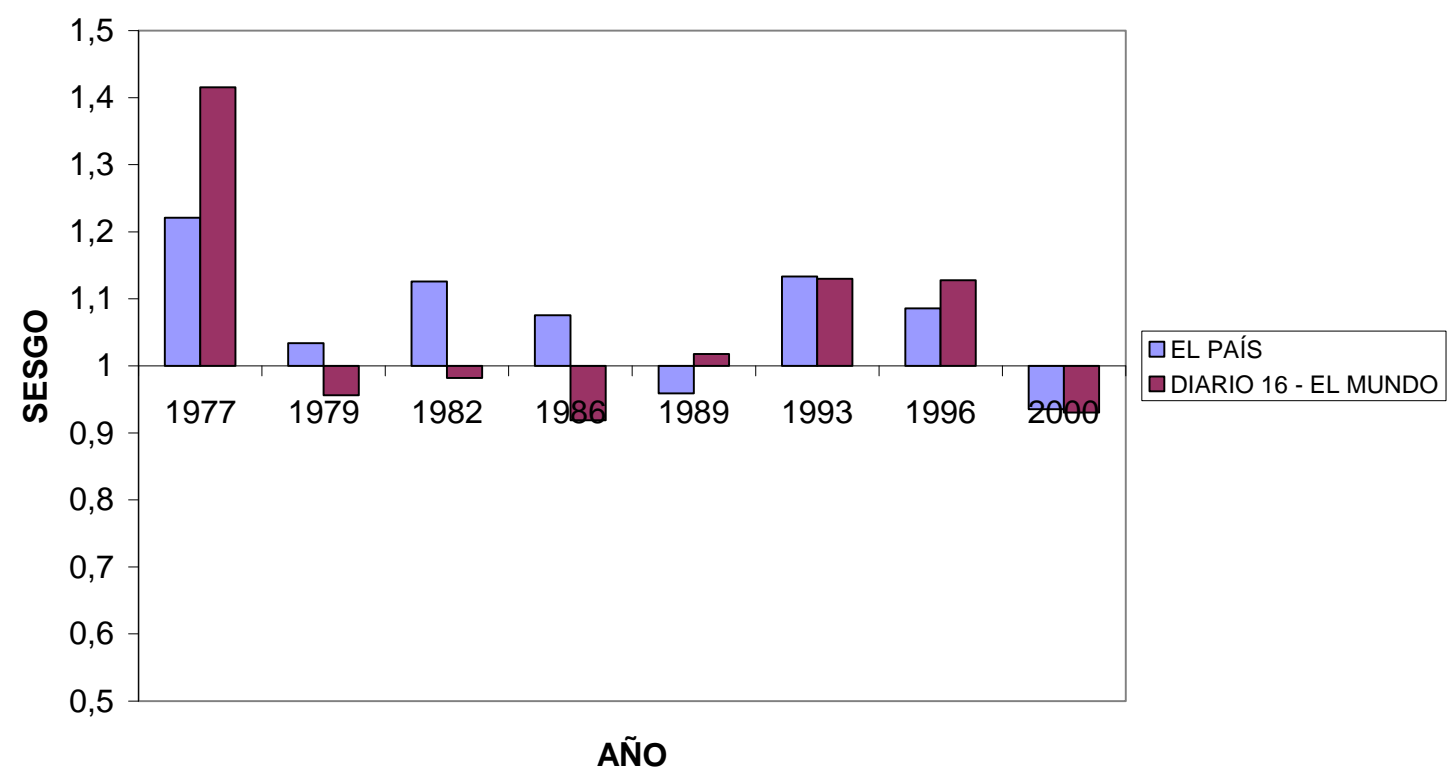

En El País, curiosamente, se infravalora durante este periodo la intención de voto hacia el partido que, comúnmente, se cree que es el que defiende: el PSOE. Esta estrategia tiene como objetivo el estímulo del voto, en la misma línea que la propaganda negativa que comenzó a utilizar el PSOE en los 90. Entre 1993 y 1996, la estimación de voto infravalora la opción del PSOE, mal visto socialmente por los casos de corrupción, que fomenta el enmascaramiento del voto hacia este partido.

Por el contrario, en el año 2000, el PSOE es sobrevalorado por la tendencia de las encuestas anteriores y porque la inercia del entendimiento del sistema político fomenta la concepción del mantenimiento del statu quo. 


\subsection{Sesgo de la intención de voto al PP}

El Partido Popular ha ido creciendo al tiempo que la democracia española. En los primeros momentos, era simplemente un partido minoritario, poco atractivo para el electorado por su pasado franquista. A medida que el partido y la sociedad española cambian, su posición en las encuestas de opinión varía.

EVOLUCIÓN SESGOS AP-PP

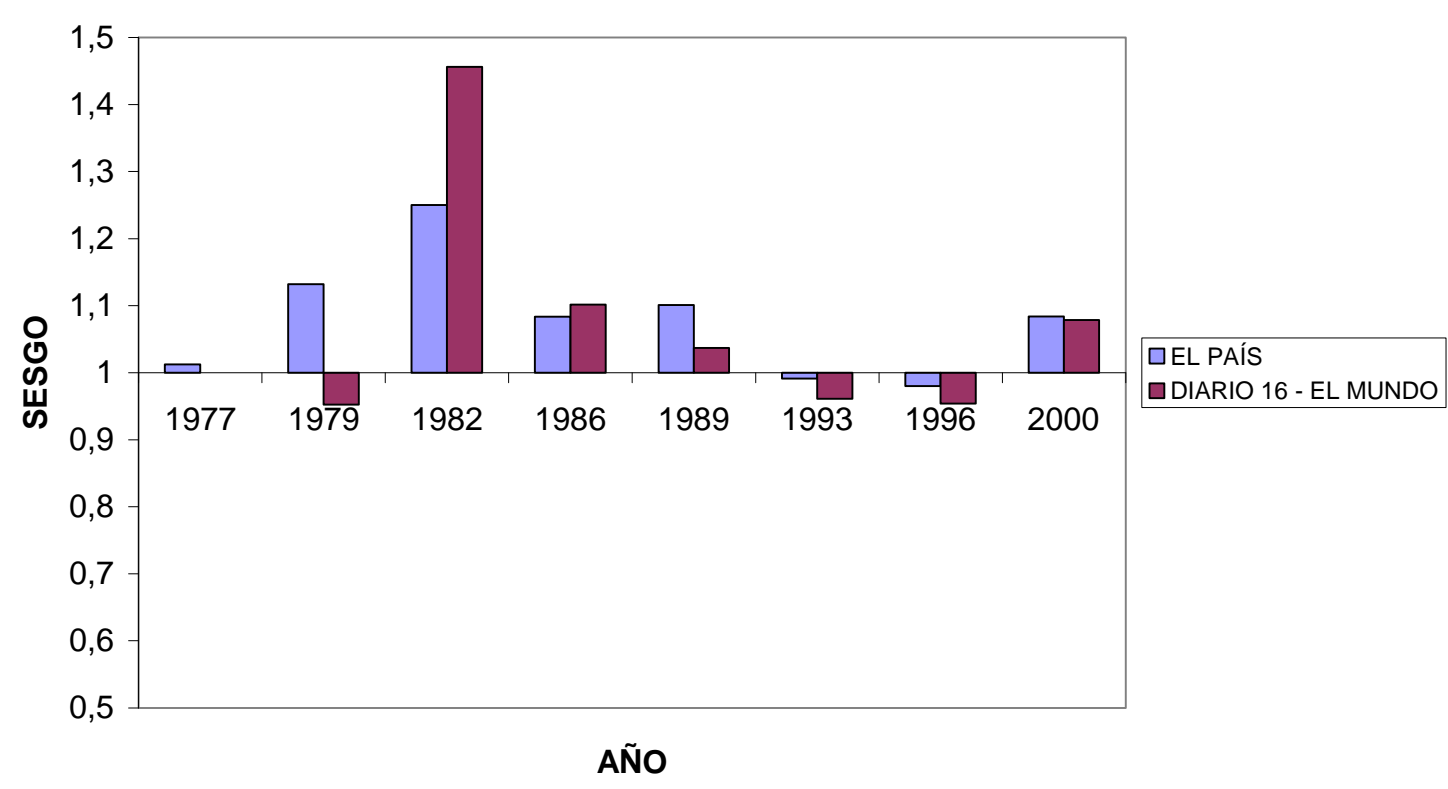

En los años 80, es infravalorado el PP y hasta los años 90, este partido no cobra su importancia en los sondeos, debido a su mala imagen por estar relacionado con el régimen anterior. En los años 90, por el contrario, el PP comienza a ser la alternativa a un sistema que cada vez se configura más como bipartidista y las encuestas le sobrevaloran como el cambio de Gobierno esperado, pero que realmente no llegó hasta un tiempo después, en el año 1996, cuando consiguió la mayoría simple para gobernar. En el año 2000, sus resultados son infravalorados, porque aún la inercia de lo presupuesto es más fuerte que la imagen de los datos de las encuestas. 


\section{Conclusiones}

La evolución a lo largo del tiempo de la encuestas de intención de voto evidencia un mayor ajuste 0 acierto con respecto a los resultados finales, pese a las críticas registradas en los medios de comunicación en contra de este sistema de estimación.

Se constata además que los prejuicios políticos, en cada momento, pueden influir directamente en la interpretación de los datos obtenidos a través de una encuesta, ya que condicionan los índices de corrección. Como se puede comprobar, inicialmente se infravalora la potencialidad del PSOE y posteriormente del PP, y, finalmente, se cambian las tornas, siempre dirigidos por ideas prefijadas de los medios de comunicación.

En caso de que las encuestas ofrezcan resultados de "empate técnico", los diarios apuestan por dar una interpretación favorable a lo esperable por parte de unos medios u otros, como ocurrió en el año 1993.

\section{Bibliográfia}

BUDGE, I. y FARLIE, D.J. (1986): Pronósticos electorales: puntos de debate y estrategia política. Centro de Estudios Constitucionales. Madrid.

CAPO, J. (1998): Las encuestas electorales en la sociedad mediática. Centro de Estudios Políticos y Constitucionales. Madrid.

COTARELO, R. (1996): El alarido ronco del ganador: las elecciones de 1996, los medios de comunicación y el porvenir de España. Grijalbo. Barcelona.

CRESPI, I. (1988): Pre-election polling: sources of accuracy and error. Rusell Sage Foundation. New York. 
CRUZ, A. (1993): Comunicación política y elecciones en España 1975-1991. Editorial de la Universidad Complutense. Madrid.

ESTRUCH, J. (2000): Historia Oculta del PCE. Editorial Temas de Hoy. Historia. Barcelona.

FERNÁNDEZ, J. O. (1994): Diseño y utilidad de las encuestas preelectorales. Servicio Central de Publicaciones del Gobierno Vasco. Vitoria-Gasteiz.

MARTIN, J. L., MARTINEZ-SHAW, C. y TUSELL, J. (1998): Historia de España. Editorial Taurus.

MONZÓN, C. (2005): Encuestas y elecciones. Editorial Tecnos Ensayos. Madrid.

MOOD, A. M. (1955): Introducción a la teoría de la estadística. Editorial Aguilar. Madrid.

OÑATE, P. (1999): Análisis de datos electorales. Centro de Investigaciones sociológicas. Madrid.

PREGO, V. (1999): Diccionario de la transición. Editorial Plaza y Janes. Barcelona.

PREGO, V. (2000): Presidentes. Editorial Plaza y Janes. Barcelona.

KIRKPATRICK, S. A. (ed.) (1976): American electoral behavior. Sage. London.

TUSELL, Xavier (1975): La España del siglo XX. Desde la muerte de Alfonso XIII a la de Carrero Blanco. Dopesa. Barcelona.

YOUNG, M. L. (1987): The american dictionary of campaigns and elections. Hamilton Lanham.

\section{Hemerografía}

Diario 16 (1977-1986).

El Mundo (1989-2000).

El País (1977-2000).

$A B C$ (1975-2000). 


\section{Webgrafía}

Radio Directory. Disponible en: www.radiodirectory.com/. Consultado el 16-05-2006. Colpisa. Disponible en: www.colpisa.com/NOSUSCRIPTOR/index.html. Consultado el 17-05-2006.

Europa Press. Disponible en: www.europapress.es/europa/portada.asp. Consultado el 17-05-2006.

Informativos T5. Disponible en: www.informativost5.com/. Consultado el 18-052006.

RTVE. Disponible en: www.rtve.es/. Consultado el 01-06-2006.

RTVV. Disponible en: www.rtvv.es/. Consultado el 02-06-2006.

Vanguardia. Disponible en: www.vanguardia.es/. Consultado el 02-06-2006.

$A B C$. Disponible en: www.abc.es/. Consultado el 16-06-2006.

El Mundo. Disponible en: www.el-mundo.es/. Consultado el 19-05-2006.

El País. Disponible en: www.elpais.es/ Consultado el 18-06-2006.

La Radio Digital. Disponible en: www.laradiodigital.com/ Consultado el 18-04-2006.

España siglo XX. Disponible en: http://vespito.net/historia/transi/. Consultado el 1704-2006.

La transición española. Disponible en: www.salman-psl.com/la-transicionespa\% f1ola/index.html. Consultado el 15-06-2006.

Demoscopia. Disponible en: www.demoscopia.com. Consultado el 15-06-2006.

Sigma. Disponible en: www.sigmadoscgt.org/. Consultado el 15-06-2006. 